\title{
La metodología de proyecto social en la educación superior: una oportunidad para armonizar el proyecto de vida con el proyecto de sociedad
}

\author{
SONIA BRITO RODRÍGUEZ \\ Universidad Autónoma de Chile - Chile \\ LORENA BASUALTO PORRA* \\ Universidad Católica Silva Henríquez - Chile \\ LUIS REYES OCHOA ${ }^{* * *}$ \\ Universidad Católica Silva Henríquez - Chile \\ Recibido el 10-08-18; evaluado el 24-12-20; \\ aceptado el 18-02-21
}

\begin{abstract}
RESUMEN
El artículo presenta los resultados de una investigación cualitativa referida a la evaluación de la metodología de proyecto social inicial, experiencia de aprendizaje ejecutada por los y las estudiantes en el marco de las actividades curriculares del plan transversal de la Universidad Católica Silva Henríquez (UCSH).
\end{abstract}

\footnotetext{
* Doctora en Ciencias de la Educación, mención educación intercultural, Universidad de Santiago de Chile. Magíster en Educación Superior, Universidad Andrés Bello, Santiago Chile. Trabajadora social, Universidad Tecnológica Metropolitana. Actualmente es académica-investigadora, Escuela de Trabajo social, Universidad Autónoma de Chile. Directora del Grupo de Investigación Género y Sexualidades en contextos de diversidades culturales. Correo electrónico: sonia.brito@uautonoma.cl. https://orcid. org/0000-0002-1211-1125

** Magíster en Educación, Universidad Andrés Bello, Chile. Magíster en Teología Dogmática, Pontificia Universidad Católica de Chile. Licenciada en Ciencias Religiosas, Pontificia Universidad Católica de Valparaíso. Profesora de Religión y Moral, Pontificia Universidad Católica de Valparaíso. Actualmente se desarrolla como académica del Instituto teológico Egidio Viganó, Universidad Católica Silva Henríquez, Santiago de Chile. Correo electrónico: lbasualtop@ucsh.cl. https://orcid.org/0000-0001-8780-0841

*** Doctor en Didáctica y Organización Educativa, Universidad de Sevilla. Magíster en Educación con mención Curriculum Educacional, Universidad Metropolitana de Ciencias de la Educación. Profesor de Educación Religiosa para la Enseńanza Media, IPES Blas Cañas. Actualmente se desarrolla como académico permanente, Instituto Interdisciplinario en Pedagogía y Educación (IIPE), Facultad de Educación, Universidad Católica Silva Henríquez (UCSH) Correo electrónico: 1reyeso@ucsh.cl. https://orcid.org/0000-0001-6070-6169
} 
Esta metodología, permite vincular el proyecto de vida de los jóvenes con el proyecto de sociedad, es decir, es una estrategia que se realiza en terreno, aportando desde el primer ańo de universidad al sentido de una formación profesional que contribuya a la construcción de una sociedad más justa y fraterna. El artículo plantea la necesidad de validar, a través de una estrategia evaluativa, esta metodología activa e innovadora, que permite aprendizajes significativos en el ámbito de la educación superior.

Palabras claves: Métodos de enseñanza, responsabilidad, formación integral, aprendizaje situado, evaluación.

\section{The methodology of social project in higher education: an opportunity to harmonize life project and society project}

\section{Abstract}

The article presents the results of a qualitative research referred to the evaluation of the initial social project methodology, learning experience executed by the students within the framework of the curricular activities of the transversal plan of the Catholic University Silva Henriquez (UCSH). This methodology allows linking the life project of young people with the society project, that is, it is a strategy that is carried out in the field, contributing from the first year of university to the sense of a professional training that contributes to the construction of a more fair and fraternal society. The article raises the need to validate, through an evaluative strategy, this active and innovative methodology that allows significant learning in the field of higher education

Keywords: Teaching methods, responsibility, integral training, situated learning, evaluation.

\section{A metodologia do projeto social no ensino superior: uma oportunidade para harmonizar projeto de vida e projeto de sociedade}

\section{RESUMO}

O artigo apresenta os resultados de uma pesquisa qualitativa referente à avaliaçáo da metodologia de projeto social inicial, experiência de aprendizagem realizada pelos alunos e alunas no âmbito das atividades curriculares do plano transversal da Universidade Católica Silva Henríquez (UCSH). Essa metodologia permite vincular o projeto de vida dos jovens ao projeto da sociedade, ou seja, é uma estratégia que se desenvolve no campo, contribuindo desde o primeiro ano de universidade ao sentido de formação profissional que auxilie à construção de uma sociedade mais justa e fraterna. $\mathrm{O}$ artigo suscita a necessidade de validar, por meio de uma estratégia avaliativa, essa metodologia ativa e inovadora que possibilite aprendizagens significativas no campo da educação superior.

Palavras-chave: métodos de ensino, responsabilidade, treinamento integral, aprendizagem situada, avaliação. 


\section{INTRODUCCIÓN}

En el marco de la renovación curricular de la educación superior, las universidades han debido incorporar nuevas metodologías y didácticas activas, participativas e innovadoras que van promoviendo un aprendizaje centrado en los y las estudiantes. Esto, con el propósito de renovar las técnicas tradicionales de la lección magistral, el aprendizaje memorístico y la repetición mecánica de contenidos (Zabalza, 2007), de modo de transitar hacia una innovación pedagógica universitaria que busca potenciar las habilidades del alumnado a través del desarrollo y mejora de las prácticas pedagógicas (Leal, 2015).

La necesidad de transformar las metodologías de educación superior surge desde la renovación curricular y didáctica impulsada por el proyecto Tuning que propicia la relación permanente de la formación universitaria con la sociedad civil, valorando el acercamiento temprano al mundo laboral como forma de aprendizaje significativo (Proyecto Tuning América Latina, 2007).

$\mathrm{Al}$ respecto, en los últimos años, se puede apreciar el interés de las universidades por aplicar metodologías situadas que permiten al estudiante tener un acercamiento temprano al contexto, aplicando sus conocimientos a situaciones reales y promoviendo en ellos el carácter social de la profesión. En este proceso, las metodologías de enseñanza y la evaluación de los aprendizajes se tornan altamente significativos en el proceso formativo, por lo que, es imperioso seleccionar aquellas estrategias que alcanzan mejores resultados. De esta manera, diferentes tipos de estrategias en terreno han demostrado sus beneficios en la educación superior (Tapia, 2006; Martínez, 2008; Puig, 2009; García y Rubio, 2010; Batlle, 2013; Santos, Sotelino y Lorenzo, 2015; Rodríguez 2020). Dichos autores y autoras coinciden que, junto con una óptima planificación, lo fundamental es descubrir el impacto en la enseñanza, por lo que es imprescindible contar con instrumentos evaluativos validados que permitan una retroalimentación constante (Álvarez, Martínez, González y Buenestado, 2017; Bueno y García, 2014; Borgobello, Peralta y Roselli 2010; Tünnermann, 2006; Méndez y Peña, 2017; Peralta y Marín, 2020).

De este modo, el artículo tiene como propósito aportar a la búsqueda de mejores metodologías activas, participativas e innovadoras en la educación superior proponiendo una forma de evaluación y análisis de la estrategia, a través del testimonio de estudiantes y cuerpo académico.

Esta investigación se realiza en la $\mathrm{UCSH}^{1}$, específicamente, en el Plan de Formación Integral (PFI) cuya misión es contribuir a la formación general,

\footnotetext{
Institución de educación universitaria privada de Santiago de Chile perteneciente a la congregación Salesiana.
} 
tributando a las competencias genéricas institucionales. El PFI tiene como objetivo plasmar el sello identitario institucional, referido al compromiso con la justicia y el bien común desde una pedagogía del acompañamiento y de la mediación cultural (UCSH, 2014a).

Durante el primer ańo académico, el PFI programa el curso de Desarrollo Personal donde se despliega la metodología de Proyecto Social Inicial (PSI). El PSI tiene como propósito que los y las estudiantes vinculen su proyecto de vida con su proyecto de sociedad contribuyendo al perfil de egreso que, curricularmente, se desarrollan a través de las competencias genéricas institucionales.

El artículo presenta las sistematizaciones de las evaluaciones de los PSI desde una metodología cualitativa a través del análisis de los relatos de estudiantes y cuerpo académico recogidos mediante grupos de discusión, fichas de proyectos sociales y testimonios, los cuales se han registrado durante diez ańos. Para el presente estudio, se seleccionaron las sistematizaciones de los años 2014 a 2018 puesto que, en este período, la Universidad inicia su tránsito hacia un currículum basado en competencias. Los relatos se analizan de acuerdo con las competencias genéricas institucionales que se dividen en identitarias y genéricas (UCSH, 2014b). El objetivo de este análisis es evidenciar si el PSI es una metodología que posibilita la integración de las dimensiones personales y sociales de los y las universitarias reconociendo, a través de ella, el valor intrínseco de la profesión como responsabilidad social hacia la comunidad.

El documento se ordena en cuatro apartados. El primero se refiere al marco teórico, donde se instala la discusión de la necesidad de renovar las metodologías en la educación superior; el segundo presenta la metodología de investigación; en el tercero se exponen los resultados a través de las experiencias del estudiantado y el cuerpo académico, usando como criterio las competencias genéricas institucionales y, finalmente, en el cuarto se despliega la discusión a partir de los resultados.

\section{Marco teórico}

\subsection{Desafíos metodológicos de la educación superior}

En las últimas décadas una de las transformaciones más importantes que ha ocurrido en Chile con respecto a la educación superior es la masificación de la cobertura que alcanza a un nuevo sujeto educativo, en tanto jóvenes provenientes de estratos sociales más vulnerables. Como lo señalan Jiménez y Lagos (2011), este nuevo estudiante se caracteriza por ser la primera generación de su familia en ingresar a la universidad y, por ende, sus capital económico, 
social y cultural suele ser de menor nivel frente a estudiantes clásicos o herederos. Según Jiménez y Lagos (2011) es necesario que la educación superior alcance una «segunda generación de reformas» (p. 11), una de ellas es superar los métodos anticuados de enseńanza-aprendizaje, actualizando y renovando el curriculum. Como señala García (2018) la docencia universitaria necesita redefinirse, transitando desde una pedagogía más bien tradicional, a una docencia flexible, interconectada y diversa; pues el actual escenario cultural requiere de una enseñanza activa y un aprendizaje conectado a los soportes tecnológicos y nuevas plataformas. De hecho, la educación desde la pandemia ha tenido que transitar hacia el mundo virtual descubriendo su dificultades y posibilidades, lo cual ha demandado descubrir nuevas formas de enseñanzaaprendizaje (Brito, Basualto, Flores y Lizana, 2020).

Es por este motivo que la innovación en educación superior es una tarea imperiosa en nuestro tiempo, en vistas a las demandas del nuevo sujeto educativo que asiste a la universidad. La innovación es entendida como un proceso integral que se incorpora tanto al diseño como al desarrollo del currículum pero que, también, involucra cambios en las prácticas pedagógicas de docentes (Leal, 2015). En este último aspecto, se focaliza el artículo considerando que la innovación en el ámbito de la docencia requiere, según Zabalza (2012), de tres condiciones: apertura de los académicos hacia la flexibilidad y adaptación, actualización de las formas de enseñanza y mejora en los procesos evaluativos.

$\mathrm{Al}$ respecto, las universidades han favorecido la búsqueda de métodos que les permitan alcanzar aprendizajes integrales a sus estudiantes, asumiendo que no solamente deben aprender habilidades cognitivas, sino, además, procedimentales y actitudinales (Brito y Reyes, 2017). Esto permite revertir la orientación academicista de la educación superior y apostar por un aprendizaje que transforme al propio estudiante en protagonista de su propia formación y, además, que no pierda su relación con el contexto social. En este sentido, las universidades adquieren una gran responsabilidad social en cuanto a formar profesionales que, no solo utilicen el saber para su beneficio particular, sino que, sobre todo, están llamados a aportar con su formación y experticia al bien común y a la construcción de la sociedad.

Además, si se considera que el proceso de enseñanza es una construcción social, el hecho de aprender con otros se realiza no solo al interior de la academia, sino que también se aprende con un alter que forma parte de la red social del estudiante. Por lo tanto, el desarrollo del PSI es un aprendizaje situado que resulta muy valioso y enriquecedor, en el sentido que los y las universitarias aplican y comunican su propio aprendizaje, activando las habilidades cognitivas, procedimentales y actitudinales (Delors, 1996). 


\subsection{La metodología educativa de Proyecto Social Inicial}

La metodología de PSI forma parte de las opciones pedagógicas donde el aprendizaje se comprende como una mediación social a la luz de las teorías de Vygotsky y el aporte de Dewey (Pozo, 2010) quienes sostienen que, una verdadera educación, es aquella que está basada en la experiencia de interrelación con el medio (Santos, Sotelino, Lorenzo, 2015). El PSI se inserta en el área de metodologías que busca desarrollar el proceso de enseñanza-aprendizaje mediante el servicio (Pimienta, 2012), enfatizando la educación ética a través de la acción (Batlle, 2013).

La metodología de PSI es una de las estrategias de aprendizaje declaradas por el PFI y se le define como "formas de acercamiento a la realidad social a través de actividades de coordinación y trabajo colaborativo en entornos socialmente desfavorecidos» (UCSH, 2016, p. 32). De esta manera, los y las estudiantes la despliegan en la actividad curricular de Desarrollo personal cursada en el primer año, a modo de una primera experiencia de aprendizaje situado.

La actividad curricular busca apoyar en el discernimiento de la elección de su carrera. Se inicia con una primera unidad donde se realiza una revisión de su proyecto de vida buscando los hitos relevantes que permiten rescatar personas, situaciones y lugares significativos que contribuyeron a su elección profesional.

Para tratar esta temática, se priorizan las metodologías activas, participativas e innovadoras que permitan potenciar el despliegue de habilidades y destrezas de estudiantes que aporten a su desarrollo personal, profesional y perfil de egreso. Se busca que los aprendizajes se enfoquen en la percepción de sí mismos y de otros, valorando su autonomía, corporalidad y autocuidado, trascendencia y diversidad, para así, resignificar su proyecto de vida personal, familiar y profesional, focalizando su quehacer socialmente responsable. En el proyecto de vida se solicita a los y las estudiantes que elaboren un relato de su historia de vida, que contenga cuatro hitos fundamentales que resulten significativos en la elección de su carrera. Además, deben elaborar una narrativa que dé cuenta de sus aspiraciones y proyecciones profesionales que considere la dimensión social y trascendente. Finalmente, comparten su proyecto de vida con el curso a través de una técnica creativa que favorezca el diálogo y el aprendizaje con el curso (Musre, 2015).

Llegado a esta parte de la metodología, se les propone trabajar colaborativamente con sus pares en el PSI vinculando su proyecto de vida con un proyecto de sociedad, el cual se concreta en una propuesta que les permita aproximarse a alguna problemática social. En esta instancia realizan un diagnóstico general, 
a través de una revisión bibliográfica y un diagnóstico especifico construido en la primera aproximación al terreno (In situ). Esta iniciativa se planifica, se implementa, se ejecuta y se evalúa, ello implica tener la experiencia de un aprendizaje en un escenario auténtico que favorece la comprensión por parte del estudiante de su experiencia de vida y formativa en su realidad cotidiana.

Para la realización del proyecto los y las alumnas se vinculan con instituciones de diversa índole tales como: colegios, jardines infantiles hogares de niños/nińas y de adultos mayores, junta de vecinos, corporaciones, fundaciones, gendarmerías, hospitales, personas en situación de calle, apoyo a organizaciones sociales y locales, entre otras.

\section{Metodología}

La metodología cualitativa que se utiliza para sistematizar las experiencias del PSI es de tipo hermenéutica, porque interesa rescatar el mundo de la vida expresada por los propios protagonistas, en este caso, cuerpo académico y estudiantes. El foco de interés o categorías de análisis son las competencias genéricas institucionales para así dar cuenta del desarrollo de las habilidades cognitivas, procedimentales y actitudinales obtenidas por los y las estudiantes. Es preciso aclarar que las competencias genéricas institucionales han sido definidas por la universidad como «atributos comunes a cualquier profesión para el desempeño laboral, como saberes que se plantean en términos formativos transversales [...] orientados al desarrollo integral del estudiante en concordancia con el sello identitario» (UCSH, 2014a, p. 26). Además, es necesario considerar que las competencias genéricas institucionales, operan como puente pedagógico para una formación en valores, puesto que «en un curriculum basado en competencias, los valores están anclados en el proceso educativo y se evidencian a través del despliegue de dichas competencias» (UCSH, 2017, p. 19).

Se utiliza como fuente de indagación, las conclusiones de la ficha de proyecto social (FPS), testimonios de estudiantes (TE) y testimonios de académicos y académicas (TA). La FPS es un instrumento utilizado en la actividad curricular de Desarrollo personal, donde el alumnado debe realizar un diagnóstico, plantear objetivos y construir un plan de acción para aproximarse a algún problema social. Para ello, deben realizar colegiadamente una revisión documental y un acercamiento a la realidad social para conocer en terreno las necesidades de las diversas instituciones o campos de intervención y, una vez finalizado el proyecto, los estudiantes realizan su propia síntesis evaluativa de los proyectos ejecutados (Núñez, 2016). La FPSI tiene el siguiente formato: 
Figura 1. Ficha de Proyecto Social (FPS)

\begin{tabular}{|l|l|}
\hline Nombre del proyecto & \\
\hline Fecha de realización & \\
\hline Lugar & \\
\hline Actividad curricular & \\
\hline Docente & \\
\hline Sección & \\
\hline Diagnóstico de la situación & \\
\hline Objetivo general & \\
\hline Objetivos específico & \\
\hline Procedimiento & \\
\hline Actividades y responsables & \\
\hline Recursos financieros & \\
\hline Síntesis evaluativa delproyecto ejecutado & \\
& \\
\hline
\end{tabular}

Fuente: Núñez, 2016, pp. 39-40.

En esta investigación se analizan las FPS entregadas por estudiantes durante los años 2014 a 2018, especialmente, aquella parte referida a la síntesis evaluativa del proyecto ejecutado. Cabe precisar que por cada actividad curricular se elabora una FPS, por lo tanto, se trata de un universo de doscientas fichas a lo largo de diez semestres. Sin embargo, para esta investigación se seleccionaron cincuenta, correspondientes a una en cada semestre elegida de las cinco temáticas de la actividad curricular de Desarrollo personal (Liderazgo y trabajo colaborativo, Comunicación efectiva, Afectividad y sexualidad, Prevención y autocuidado y, Derechos humanos y ciudadanía).

A su vez, durante cinco años se recogieron los testimonios de estudiantes, a través de entrevistas grupales de las cuales se seleccionaron casos representativos de cinco estudiantes que cursaron las actividades curriculares de Desarrollo personal, por lo tanto, se trabajó con cincuenta casos, organizados en diez entrevistas grupales. El guion de preguntas que se trabajó con el alumnado una vez finalizado el curso se refiere principalmente a: ¿Qué les impactó de la realización del proyecto? ¿Cómo llegaron a casa, con quienes compartieron su experiencia? ¿Creen que el tema de este curso y el realizar este proyecto les cambia la perspectiva de la profesión y de su persona? ¿Con qué aprendizaje se quedan? (Núné, 2016).

Por su parte, se trabajó con ocho académicos y académicas, por un tiempo de tres años, realizando seis entrevistas en total a través de las cuales compartieron el ejercicio del proceso de enseńanza-aprendizaje alcanzados mediante el PSI. 
El guion de preguntas que se trabajó durante las entrevistas grupales una vez finalizado el semestre se refiere principalmente a innovación de didácticas y técnicas de enseñanza-aprendizaje, despliegue de competencias genéricas, problemas sociales abordados, principales dificultades y estrategias para resolver, trabajo colaborativo con colegas, principales aprendizajes, hallazgos, proyecciones y desafíos.

En síntesis, la investigación da cuenta del análisis de cincuenta FPS que en la presentación de los resultados se numeran del 1 al 50; las diez entrevistas a estudiantes que constituye el documento TE, el cual se enumera del 1 al 10; $y$, las seis entrevistas a académicas y académicos que constituye el documento TA que se enumera del 1 al 6 .

El análisis de resultado se realiza haciendo una triangulación entre las competencias genéricas institucionales tanto identitarias y comunes, con la ficha de proyecto social (FPS), testimonios de estudiantes (TE) y testimonios de académicos y académicas (TA), teniendo presente el siguiente esquema:

\section{Figura 2. Competencias genéricas institucionales}

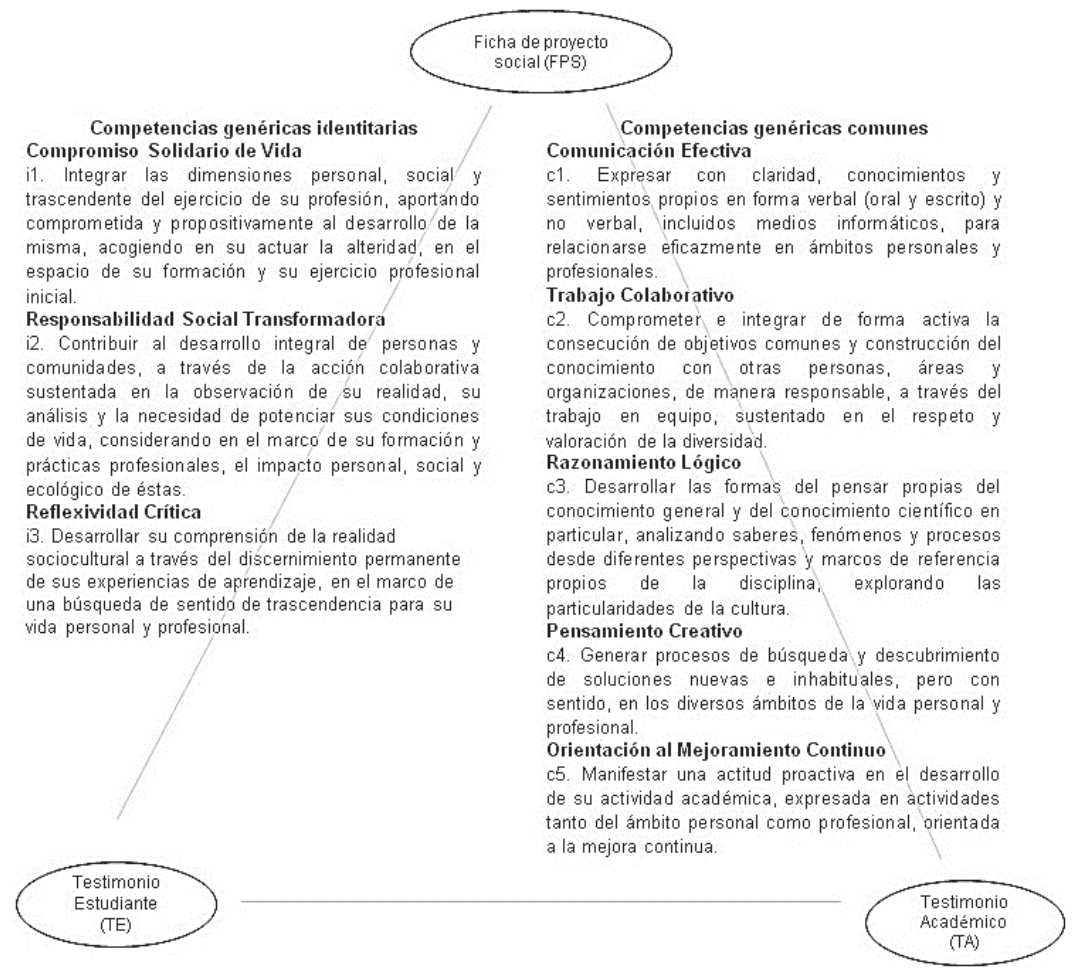

Fuente: Universidad Católica Silva Henríquez (UCSH), 2017, pp. 19-20 
La técnica de análisis comprendió cuatro etapas: (1) preanálisis, fase en la cual los relatos se organizaron según las ocho competencias genéricas declaradas por la universidad (2) Categorización, en la que se organizó y clasificó la información según los conceptos clave de cada una de las competencias según la siguiente tabla:

Figura 3. Tabla de análisis de contenido de Proyecto Social Inicial (PSI), Testimonios de Estudiantes (TE) y Testimonios de Académicos y Académicas (TA)

\begin{tabular}{|l|l|l|l|l|}
\hline \multicolumn{1}{|c|}{ Competencias } & \multicolumn{1}{|c|}{ Conceptos claves } & $\mathbf{n}^{\circ}$ FPS & $\mathbf{n}^{\circ} \mathrm{TE}$ & $\mathbf{n}^{\circ} \mathrm{TA}$ \\
\hline \multirow{4}{*}{$\begin{array}{l}\text { Compromiso solidario } \\
\text { de vida }\end{array}$} & Solidaridad & & & \\
\hline & Alteridad & & & \\
\hline & $\begin{array}{l}\text { Integración en la dimensión profesional de lo } \\
\text { personal, social y trascendente }\end{array}$ & & & \\
\hline \multirow{2}{*}{$\begin{array}{l}\text { Responsabilidad social } \\
\text { transformadora }\end{array}$} & Responsabilidad con la sociedad & & & \\
\hline & Observación y análisis de la realidad & & & \\
\hline & Potenciar condiciones de vida de otros & & & \\
\hline \multirow{2}{*}{ Reflexividad critica } & Discernimiento de sus experiencias de aprendizaje & & & \\
\hline & Búsqueda del sentido de trascendencia & & & \\
\hline \multirow{2}{*}{ Comunicación efectiva } & Asertividad & Utilización de lenguaje verbal, escrito, no verbal \\
e informático & & & \\
\hline \multirow{2}{*}{ Trabajo colaborativo } & Trabajo en equipo & & & \\
\hline & Respeto y valoración de la diversidad & & & \\
\hline \multirow{2}{*}{ Razonamiento lógico } & Análisis de saberes, fenómenos y procesos & & & \\
\hline & Explorar particularidades de la cultura & & & \\
\hline Pensamiento creativo & Búsqueda y descubrimiento de nuevas soluciones & & & \\
\hline \multirow{2}{*}{$\begin{array}{l}\text { Orientación al } \\
\text { mejoramiento continuo }\end{array}$} & Actitud proactiva & & & \\
\hline & Mejora continua & & & \\
\hline
\end{tabular}

Fuente: elaboración propia desde las competencias genéricas identitarias, Universidad Católica Silva Henríquez (UCSH), 2017, pp. 19-20

(3) análisis de las unidades de relato según competencia y conceptos clave (4) sistematización de resultados donde se seleccionan los textos más significativos para dar cuenta de cada una de las competencias.

\section{Resultados}

\section{Sistematización de Ficha de Proyecto Social (FPS), Testimonios de Estudiantes (TE) y Testimonios de Académicos y Académicas (TA)}

Coherente con el discurso institucional (UCSH, 2014a), el PSI, se evalúa y sistematiza para su mejora continua tanto en sus estrategias didácticas y evaluativas, 
como también en el rol del académico y académica y del estudiante, además de indagar el impacto de la formación en tanto si desarrollan o no las habilidades declaradas por la competencia. Esto demanda comprometer a los sujetos educativos en una permanente reflexión en la acción.

En este apartado se presenta el análisis que surge desde las competencias genéricas institucionales presentadas en la figura 2 y las percepciones de estudiantes, académicos y académicas con respecto a la experiencia del PSI, según el análisis realizado de acuerdo con la Figura 3 y a la sistematización de los resultados.

En primer lugar, se muestra el análisis desarrollado en la etapa dos. En la tabla se puede percibir que en los relatos de estudiantes, académicas y académicos se hace referencia a los distintos aspectos de las competencias genéricas:

Figura 4. Tabla de resultados de análisis de contenido de Ficha de Proyecto Social (FPS), Testimonios de Estudiantes (TE) y Testimonios de Académicos y Académicas (TA)

\begin{tabular}{|c|c|c|c|c|}
\hline Competencias & Conceptos claves & $\mathrm{n}^{\circ}$ FPS & $n^{\circ} \mathrm{TE}$ & $\mathrm{n}^{\circ} \mathrm{TA}$ \\
\hline \multirow{3}{*}{$\begin{array}{l}\text { Compromiso solidario } \\
\text { de vida }\end{array}$} & Solidaridad & $\begin{array}{l}2 ; 3 ; 5 ; 10 ; 15 ; 22 ; \\
31 ; 34 ; 44 ; 49\end{array}$ & $5 ; 3$ & 1 \\
\hline & Alteridad & $4 ; 7 ; 29$ & 2 & 4 \\
\hline & $\begin{array}{l}\text { Integración en la dimensión } \\
\text { profesional de lo personal, social y } \\
\text { trascendente }\end{array}$ & $11 ; 28$ & 1 & 4 \\
\hline \multirow{3}{*}{$\begin{array}{l}\text { Responsabilidad social } \\
\text { transformadora }\end{array}$} & Responsabilidad con la sociedad & $6 ; 7 ; 10 ; 18 ; 25 ; 30$ & $3 ; 9 ; 10$ & 5 \\
\hline & Observación y análisis de la realidad & $\begin{array}{l}1 ; 4 ; 8 ; 15 ; 21 \\
34 ; 40\end{array}$ & $4 ; 7$ & 2 \\
\hline & Potenciar condiciones de vida de otros & $8 ; 18 ; 33$ & 4 & 3 \\
\hline \multirow[t]{2}{*}{ Refiexividad crítica } & $\begin{array}{l}\text { Discernimiento de sus experiencias de } \\
\text { aprendizaje }\end{array}$ & $9 ; 28 ; 32 ; 44$ & 9 & 5 \\
\hline & Búsqueda del sentido de trascendencia & $6 ; 29 ; 42$ & 1 & 6 \\
\hline \multirow[b]{2}{*}{ Comunicación efectiva } & Asertividad & $11 ; 17 ; 39$ & $3 ; 6 ; 8$ & 3 \\
\hline & $\begin{array}{l}\text { Utilización de lenguaje verbal, escrito, } \\
\text { no verbal e informático }\end{array}$ & $5 ; 13 ; 20$ & $3 ; 6$ & 6 \\
\hline \multirow[t]{2}{*}{ Trabajo colaborativo } & Trabajo en equipo & $\begin{array}{l}2 ; 4 ; 1416 ; 25 ; 29 ; \\
31 ; 35 ; 42 ; 47 \\
\end{array}$ & $\begin{array}{l}1 ; 2 ; 4 ; \\
5 ; 8 \\
\end{array}$ & $1 ; 4 ; 5$ \\
\hline & Respeto y valoración de la diversidad & $17 ; 31$ & 3 & 2 \\
\hline \multirow[t]{2}{*}{ Razonamiento lógico } & $\begin{array}{l}\text { Análisis de saberes, fenómenos y } \\
\text { procesos }\end{array}$ & $23 ; 42$ & 1 & 3 \\
\hline & Explorar particularidades de la cultura & $13 ; 23 ; 48$ & & 5 \\
\hline Pensamiento creativo & $\begin{array}{l}\begin{array}{l}\text { Búsqueda y descubrimiento de nuevas } \\
\text { soluciones }\end{array} \\
\end{array}$ & $8 ; 15 ; 27$ & $3 ; 7$ & 3 \\
\hline \multirow{2}{*}{$\begin{array}{l}\text { Orientación al } \\
\text { mejoramiento continuo }\end{array}$} & Actitud proactiva & $12 ; 35$ & 4 & 1 \\
\hline & Mejora continua & $12 ; 35$ & 7 & 1 \\
\hline
\end{tabular}


Como se puede apreciar en la tabla, es posible reconocer en FPS, TE y TA que están presentes los conceptos claves de las competencias genéricas, desde lo cual se puede inferir que hubo desarrollo de experiencias de aprendizaje. Las competencias más desarrolladas son el Compromiso solidario de vida, la Responsabilidad social transformadora y el Trabajo colaborativo. Desde el análisis de las unidades de relato se percibe el desarrollo de las habilidades a la cual tributa cada competencia genérica, para ello, se seleccionaron algunos de los relatos más significativos. Así, la competencia de Compromiso solidario de Vida que considera como elementos centrales la solidaridad, la alteridad y la integración personal, social y trascedente (UCSH, 2014b), los y las estudiantes señalaron,

Aprendimos que se puede generar solidaridad en todos los espacios donde nos desenvolvemos, y que este curso nos permite revisar nuestra vocación [...] tenemos la convicción de que queremos reconstruir un mundo más equitativo, más solidario, más amable. (FPS22)

Pudimos ir más allá de las aulas universitarias y ver la realidad concreta de un grupo de adultos mayores que ya, en su tercera edad, comparten vivencias e historias en un lugar donde reciben cuidados y la preocupación de personas y una comunidad que está pendiente de sus necesidades. (FPS4)

Tenderles la mano, conversar un rato, ellos iban a estar contentos. Yo llegué a mi casa, les conté a mi familia, se me cayeron casi las lágrimas contándole a mi hermana todo el cuento y fue súper bonito, súper lindo, muy linda experiencia. (TE1)

Es posible apreciar en los relatos el impacto de la experiencia de vida fuera del aula, que permite resignificar e iluminar su formación profesional y personal en un ambiente interdisciplinario de ayuda fraterna, contribuyendo en la construcción de una sociedad mejor. Se constata la operativización y el despliegue de la competencia, en cuanto que los y las alumnas pudieron integrar la dimensión personal, social y trascendente, ejercitando la alteridad. Se han podido situar en el lugar de otro, sobre todo de aquél que está viviendo en una situación de desventaja social.

Igualmente, la competencia de Responsabilidad social transformadora permite que los y las universitarias contribuyan al desarrollo integral de personas y comunidades, sustentada en la observación y análisis de la realidad, potenciando la vida de otros (UCSH, 2014b). Se reflexiona lo siguiente,

Hay proyectos que se desarrollan fuera de la universidad, donde se va al encuentro con alguna comunidad: un hogar, con hogares de niños, grupos de scout, con colegios, etc. Los estudiantes han ido al lugar o la gente ha venido 
a la Universidad, porque entendemos que también es un espacio que se valora súper positivamente, que podamos también mostrarles esta experiencia de la Universidad. En cualquiera de sus formas los estudiantes tienen la experiencia de poder empaparse de la realidad (TA2).

Creemos que podemos aportar desde hoy al cambio de nuestra sociedad y no esperar a ser profesionales, ya que en el presente tenemos una gama de acciones a nuestro alcance que contribuyen al bienestar de otro; si bien muchas veces nos encontramos frente a problemas grandes que afectan a la sociedad en nuestras manos está contribuir a una sociedad más justa y equitativa, valoramos las instancias generadas por la universidad que nos permiten la participación y la libertad de acción que desarrollamos (FPS18).

En los testimonios se evidencia que tanto académicas y académicos como estudiantes dan cuenta de aprendizajes situados que se refieren a comprender la realidad, abriendo la universidad a la comunidad. Se valora el trabajo con responsabilidad que logra potenciar las condiciones de vida de otros y otras, compartiendo los sueños, preocupaciones y esperanzas. Esto permite en la trayectoria formativa, aspirar a la formación integral de los y las estudiantes, contribuyendo a su protagonismo socialmente responsable, lo cual ayuda a configurar su perfil de egreso.

Del mismo modo, la competencia de Reflexividad crítica pretende desarrollar el discernimiento de las experiencias del propio aprendizaje de los y las universitarias, a través de un discernimiento informado y la búsqueda de un sentido trascendente de la profesión (UCSH, 2014b). Las principales narrativas plantean,

A veces uno, con problemas chicos, se ahoga en un vaso de agua, y las realidades de los otros son súper fuertes y ahí a uno le viene la reflexión que con los problemas chicos uno no tiene que echarse a morir, si no que aprender de ello y ver que otras personas lo pueden estar pasando más mal y eso es súper valorable también, saber las distintas realidades y compararlas con las de cada uno (TE9).

Se contempla la educación como el principal instrumento de promoción, conocimiento y defensa de los DDHH. Por tanto, desde esas instancias educativas se plantea la relevancia de los sitios de memoria en la conformación de nuestro País, como espacios educativos reales. Dimensión que la pedagogía no puede dejar fuera pues debe ir más allá de la transmisión de contenidos (FPS6).

Se vislumbra, en el primer relato, el entendimiento de los y las estudiantes de sus propios aprendizajes, pudiendo realizar el proceso de metacognición desde la experiencia obtenida a través del PSI. Además, son capaces de discernir en el contexto social, encarnado en personas y situaciones, un sentido 
de trascendencia a su futura profesión, dándole una visión nueva al servicio y a la vocación. Lo anterior es relevante en la medida que los y las universitarias observan la realidad críticamente, utilizando los aprendizajes obtenidos como herramientas de trasformación personal y social, logrando descubrir el bien intrínseco de su profesión.

La competencia de Comunicación efectiva busca formar en la asertividad para vincularse con claridad con un otro, reconociendo los diversos contextos a través de la utilización del lenguaje verbal, escrito, no verbal e informático (UCSH, 2014b). Al respecto los y las estudiantes señalan,

Trabajamos el tema de asertividad, el stress, la violencia en el pololeo... Son muchos temas que no se conversan habitualmente. A lo más por ahí con los amigos, pero no de una manera más formal, no con altura de miras, no siendo objetivos, se podría decir, como que más en la broma. Pero en el curso se da la instancia para conversar y estos temas no se conversan ni en la casa, entonces se puede sacar mucho provecho en ese sentido (TE6)

El proyecto demandó negociar y superar diferencias para alcanzar un objetivo común. Igualmente, a través de las actividades de planeación logramos llegar a consensos, cuestión que tuvo sus complejidades, pero lo logramos gracias a que estaban claras las metas. (FPS17).

La comunicación e interacción establecida dentro del aula se proyecta al espacio extra aula, mejorando de esa manera los estilos verbales, corporales y gestuales. Esto queda de manifiesto en las formas de relacionarse entre académicos, académicas, estudiantes y personas partícipes del PSI en el proceso formativo. Otro aspecto se refiere a la relación con los otros, puesto que se hacen presentes las dimensiones emotivas como constitutivas de la comunicación efectiva, de manera que el lenguaje no es solo técnico, sino que involucra a toda la persona en el hecho comunicativo.

Por su parte, la competencia de Trabajo colaborativo busca comprometer e integrar en forma activa la construcción del conocimiento con otros, sustentado en el respeto y valoración de la diversidad (UCSH, 2014b). Al respecto, los y las universitarias señalan,

La dedicación de cada uno fue destacable, pusimos la mejor disposición para realizar la actividad, tanto en terreno como para cuando necesitábamos juntar fondos y una vez logrado el propósito llegamos al acuerdo de que fue una gran satisfacción el haber dedicado tiempo a este tipo de actividades. (FPS25).

Luego de definir la tarea definimos según las habilidades de cada uno los encargados y quienes querían integrar los grupos de trabajo para poder generar estrategias e insumos para esa labor. Ahí nos dimos cuenta de que cada uno 
es más que un estudiante y que poseemos aspectos desde lo personal que poníamos a disposición para el logro de la actividad al definir grupos y encargados y con una idea común, discutiendo en sus grupos soluciones posibles ante la propuesta de trabajo que teníamos (FPS31).

$\mathrm{Al}$ analizar los relatos es posible vislumbrar las dificultades y las alternativas de soluciones trabajadas colaborativamente. Es importante mencionar que el PSI permitió a los estudiantes exteriorizar habilidades no reconocidas anteriormente y a distribuir el trabajo de acuerdo con sus capacidades e intereses, lo que facultó enfrentar la tarea con los talentos personales que cada quien fue descubriendo. Por último, se menciona que el trabajo colaborativo requiere como condición sine qua non el respeto a las diversidades tanto del curso, como de las y los actores sociales de la comunidad.

Asimismo, la competencia del Razonamiento lógico desarrolla diferentes formas de pensar considerando el conocimiento general y científico para analizar desde diferentes perspectivas las particularidades de las culturas (UCSH, 2014b). Con respecto a esta competencia, estudiantes, y cuerpo académico señalaron,

Esta experiencia contribuye en la formación de profesionales conscientes, cultos y comprometidos con un trabajo valeroso en la defensa de las personas más desprotegidas de la sociedad, promoviendo también, la visibilidad y relevancia de la memoria histórica, entre sus pares en instancia universitaria. (FPS42)

Buscamos crear espacios específicos de educación universitaria con un abordaje Socioeducativo, creativo e histórico que nos interpele como personas y como profesionales en la formación integral de «un nosotros» que abre caminos y cruza nuevas perspectivas y desafíos. Esta propuesta busca superar en parte la carencia de memoria histórica que, a pesar de los avances en estas materias, aún tenemos en Chile. (TA 5)

Desde los testimonios, se puede visualizar el compromiso con la historia y con la sociedad, analizan los fenómenos sociales y a la vez vislumbran desafíos y oportunidades de cambios. Además, se proyectan como profesionales comprometidos con la dignidad de la persona desde un reconocimiento de la memoria histórica de su país, permitiéndoles proyectarse como ciudadanos/as activos/ as. También, es posible apreciar, que los y las universitarias leen contextos y culturas, analizando a partir del acervo de aprendizajes y saberes las contingencias y complejidades de un mundo cambiante. Esto les permite visualizar mejor el contexto, los desafíos y la responsabilidad del desarrollo de su futura profesión, constituyéndose de esta manera en un verdadero aporte al país. 
Por su parte, la competencia de Pensamiento creativo insta a la búsqueda de nuevas soluciones para los diversos ámbitos de la vida personal y profesional (UCSH, 2014b). Con respecto a esta competencia los/las universitarias señalaron, lo siguiente,

Al estar nosotros un día domingo en un carnaval nos parecía increíble, ver como todo se teñía de colores y música y que nosotros estábamos ayudando a que eso pasara nos llenó de orgullo invitándonos a reflexionar sobre nuestro quehacer actual y futuro, es decir, que no solo podemos aportar desde nuestras profesiones, sino que existen espacios y escenarios distintos en donde podemos realizar acciones sociales potentes y que contribuyen al desarrollo de las personas. Como grupo nos sentimos satisfechos ya que el vivir una experiencia fuera de la Universidad nos permite conocernos de forma distinta y permite darnos cuenta de que tenemos otras habilidades que no habíamos explorado tanto a nivel personal y grupal, existiendo cosas que nos unen como las ganas de ayudar, la generación de ideas, descubrir que hacemos más cosas de las que creemos que somos capaces y que nos potenciamos positivamente (FPS15)

A partir de este relato, se puede constatar, que los y las estudiantes descubren capacidades que no habían explorado, las cuales se manifiestan cuando deben desarrollar actividades inusuales. Esto les coloca en contacto con escenarios, personas, grupos y temáticas novedosas, donde la creatividad y espontaneidad es una constante. Lo anterior, lleva a romper las estructuras cognitivas y procedimentales estereotipadas permitiendo dar un salto cualitativo a nuevas experiencias que se traducen en nuevos aprendizajes en contextos abiertos y reales. Además, los y las alumnas se atreven a explorar realidades distintas y enfrentar lo desconocido minimizando el temor y la desconfianza para situarse frente a un otro que tiene lenguajes y prácticas diferentes.

Finalmente, la competencia de Orientación al mejoramiento continuo busca promover una actitud proactiva en el desarrollo de la experiencia académica del estudiante, orientada al progreso del ámbito personal y profesional (UCSH, 2014b). Al respecto señalaron los/las estudiantes,

Es súper necesario creo yo, el tema de la planificación, de fijar los objetivos. Para todo en la vida, nos va a servir esto, no solo para una acción social, si no para planificar, no sé... un trabajo grupal. Hay que planificar, fijar los objetivos y que todos remen para el mismo lado. También en el ámbito laboral hay que hacer una planificación y también hay que recurrir a lo mismo, entonces yo creo que nos a servir para toda la vida (TE4)

Para muchos de nosotros era la primera vez que visitábamos un Hogar de niños teníamos miedos y temores asociados al no saber qué pasaría cuando llegáramos allí, sin embargo, todo resultó mejor de lo que esperábamos. Ya en terreno, 
hubo grandes aciertos como errores por corregir, que una vez sean revisadas en sesión grupal, permitirán establecer mejoras personales y grupales para futuras intervenciones (TE7).

La realidad desafía a construir espacios de aprendizajes en donde se ejercite la capacidad de flexibilidad y actitud proactiva de adelantarse a las problemáticas y contingencias, reinventando y reprogramando las actividades, haciéndolas más acordes a los contextos y propósitos trazados. Además, los y las universitarias valoran el hecho de programar las actividades, es decir, son capaces de desarrollar procesos de metacognición que les permite vincular los aprendizajes de aula con las necesidades que descubren en el entorno y de esa manera planificar sus aportes con sentido. Igualmente, valoran este tipo de actividades de intervención social, pues aportan a la mejora continua de la sociedad y se potencian competencias que son importantes para el ejercicio profesional.

\section{DisCusión}

El PFI permite desplegar y trabajar el proyecto de vida vinculado a un proyecto de sociedad, toda vez que propicia la profundización de la biografía y de la trayectoria personal del estudiante. Este entramado de subjetividades, en el marco de una formación con sentido, se inserta en una opción pedagógica que considera el proyecto de sociedad como un aspecto central en la iniciación del ejercicio profesional, que se aprende desde la vida misma. Esta articulación permite realizar un proyecto formativo situado, analizando o vislumbrando los fenómenos, los problemas y las políticas sociales. A su vez, los y las estudiantes llegan a la universidad con sus saberes previos, experiencias, biografías y sueños, encontrándose con un curriculum favorecedor de su formación integral donde se le propone articular un proyecto social al servicio de la comunidad. Al respecto el PSI se inserta en el curriculum de primer ańo de los y /las universitarias para favorecer la relación temprana entre profesión y contexto, dimensión que es fundamental al momento de transitar hacia un curriculum por competencias, según lo señalado por el Proyecto Tuning América Latina (2007).

También el PSI es una oportunidad para favorecer la responsabilidad social del estudiantado desde el inicio de su formación profesional, en ese sentido, se aproximan a territorios y personas desfavorecidas y vulneradas en su calidad de vida y bienestar. Con esta experiencia, los y las universitarias valoran el bien intrínseco de su profesión reconociéndola como un servicio que se despliega 
hacia otros, para mejorar y aportar a la sociedad. Así, aprender una profesión no significa solo incorporar un conjunto de teorías, sino más bien valorar el telos que, es justamente, el servicio que se despliega para alcanzar la transformación social.

Por su parte, si bien es cierto que las universidades están promoviendo la innovación con diversas metodologías, es fundamental que se considere el elemento evaluativo para que realmente se pueda afirmar que se está realizando una innovación docente (Peralta y Marín, 2020). En este sentido, el artículo proporciona una metodología evaluativa que considera las percepciones de estudiantes y académicas y académicos para descubrir si efectivamente las habilidades declaradas en las competencias genéricas se están desarrollando desde el primer año de formación.

Finalmente, cobra relevancia en este tipo de propuesta la figura del académico o académica quien media, facilita y acompańa los aprendizajes significativos que se encarnan en la persona del estudiante como una experiencia vital. De esta manera, las universidades que buscan innovar liberan al académico y académica del uso de metodologías tradicionales y promueven nuevas búsquedas que favorezcan el aprendizaje de estudiantes, aspecto necesario según Jiménez y Lagos (2011), si se tiene en cuenta el nuevo tipo de alumnado que ingresa a la universidad.

\section{CONCLUSIONES}

A través de la investigación cualitativa se pudo realizar el análisis de las fichas de proyecto social, testimonio de estudiantes, académicos y académicas, evidenciando el proceso de enseńanza-aprendizaje bajo la metodología PSI, a la luz de ocho competencias genéricas institucionales.

A partir de este método de evaluación se puede constatar que el PSI es una metodología integral, participativa y significativa, puesto que los mismos estudiantes dan testimonio que la estrategia permite un acercamiento temprano a la realidad social vinculando su propio proyecto de vida con su proyecto de sociedad. Además, realzan con gran entusiasmo que este proceso les posibilita relacionarse con la realidad social que les rodea, tomando conciencia del impacto que sus acciones y decisiones generan en su entorno. Se plantea de este modo, la necesidad de ir construyendo un perfil ético profesional, que aborde como pilar la noción de un/una estudiante socialmente responsable, íntegro y coherente con sus valores y sus decisiones.

Es necesario señalar, que la metodología de PSI se sustenta en un sólido fundamento teórico, es este caso, una estrecha relación entre las temáticas 
de la actividad curricular, tales como, afectividad, autoestima, habilidades interpersonales y personales, historia, proyecto de vida y opciones valóricas, elementos que, conjugados, fundamentan la experiencia práctica de servicio solidario y trabajo interdisciplinario. Lo anterior, supone la confluencia de distintas miradas, hecho que posibilita un alcance más integral de los proyectos. Es también, gracias a este proceso, que los y las estudiantes ejercitan su vocación de servicio ampliando la comprensión de su carrera, reforzando su sentido y expandiendo sus posibilidades.

Finalmente, se puede afirmar, que la metodología de PSI en la educación superior es una oportunidad de promover en los y las universitarias la armonización de su proyecto de vida con un proyecto de sociedad que tiende al bien común. Conclusión que es posible sostener ya que se utilizó un método de evaluación basado en la percepción de aprendizajes de estudiantes y cuerpo académico sobre las competencias genéricas institucionales.

\section{REFERENCIAS BIBLIOGRÁFICAS}

Álvarez, J., Martínez, M., González, H. y Buenestado, M. (2017). El aprendizaje-servicio en la formación del profesorado de las universidades españolas. Revista de Pedagogía, 75(267), 199-217. https://doi.org/10.22550/ REP75-2-2017-02

Batlle, R. (2013). El aprendizaje-servicio en España: el contagio de una revolución pedagógica necesaria. Madrid, España: PPC.

Brito, S., Basualto, L., Flores, C., Lizana, R. (2020). La escucha y la voz como instrumentos de intervención socioeducativa. En S. Brito, L. Basualto, R. Azócar, V. Gálvez y C. Flores (eds.), Intervención social y educativa en tiempos de pandemia (pp. 100-124). Santiago, Chile: Le Monde Diplomatique, Aún creemos en los sueños y Universidad Autónoma.

Brito, S. y Reyes, L. (2017). Hacia un curriculum basado en competencias. En S. Brito, L. Reyes y L. Basualto (comps.), Formación transversal y competencias genéricas en universidades (pp. 209-220). Santiago de Chile: UCSH.

Borgobello, A., Peralta, N. y Roselli, N. (2010). El estilo docente universitario en relación al tipo de clase y a la disciplina enseñada. Liberabit, 16(1), 7-16.

Bueno, T. y García, D. (2014). Hacía una nueva metodología docente. El proyecto social real aplicado al emprendimiento universitario. En M. Camacho (ed.), Procesos de aprendizajes de vanguardia en la enseñanza superior (pp. 193-210). Madrid, España: ACCI.

Delors, J. (1996). La educación encierra un tesoro. París, Francia: Unesco. 
García, R. (26 de junio de 2018). La transformación de la docencia universitaria: una invitación. Aequalis, Foro de educación superior. Recuperado de https://aequalis. cl/articulos/la-transformacion-de-la-docencia-universitaria-una-invitacion/

García, X. y Rubio, L. (2010). Prácticas de ciudadanía. Diez experiencias de aprendizaje y servicio. Barcelona: Octaedro.

Jiménez, M. y Lagos, F. (2011). Nueva geografía de la educación superior y de los estudiantes. Una cartografía del sistema chileno, su actual alumnado y sus principales tendencias. Santiago, Chile: Universidad San Sebastián.

Leal, S. (2015). Innovación a la docencia. En S. Brito y S. Leal (comps.), Innovación y transversalidad en el Plan Común Universidad. Experiencias y reflexiones de los docentes de la Universidad Católica Silva Henriquez (pp. 13-16). Santiago, Chile: UCSH.

Martínez, M. (ed.) (2008). Aprendizaje servicio y responsabilidad social de las universidades. Barcelona, España: Octaedro-ICE.

Méndez, J. y Peña, J. (2017). Sistema de medición de Competencias Genéricas en asignaturas de Formación General Electiva en estudiantes de Pregrado de la Universidad de la Frontera: propuesta metodológica como un aporte en la formación Integral. En S. Brito, L. Reyes, y L. Basualto (comps.). Formación transversal y competencias genéricas en universidades. Claves para una renovación curricular con compromiso social (pp. 33-52). Santiago de Chile, UCSH.

Musre, V. (2015). Narrativa de historia y proyecto de vida. Una herramienta para el desarrollo integral de los estuantes de la UCSH. En S. Brito y S. Leal (comps.), Innovación y transversalidad en el Plan Común Universidad, (pp. 63-84). Santiago de Chile: UCSH.

Núñez, E. (2016). El proyecto social como experiencia significativa en el desarrollo profesional. En S. Brito (comp.), Desde la pedagogía del descubrimiento a la praxis innovadora (pp. 27-47). Santiago, Chile: UCSH.

Peralta, M. y Marín, J. (2020). Tendencias de la evaluación y sus perspectivas en la educación superior. Revista Saberes APUDEP, 3(2), 1-20.

Pimienta, J. (2012). Estrategias de enseñanza-aprendizaje. Docencia universitaria basada en competencias. Ciudad de México: Pearson.

Pozo, J. (2010). Teorías cognitivas del aprendizaje. Madrid, España: Morata.

Proyecto Tuning América Latina (2007). Reflexiones y perspectivas de la educación superior en América Latina. Informe final-Proyecto Tuning-América Latina 2004-2007. Bilbao, España: Universidad de Deusto.

Puig, J. (coord.) (2009). Aprendizaje Servicio (ApS). Educación y compromiso cívico. Barcelona, España: Graó. 
Rodríguez, R. (2020). Aprendizaje Servicio y compromiso académico en Educación Superior. Revista de Psicodidáctica, 25(1), 45-51. https://doi.org/10.1016/j. psicod.2019.09.001

Santos, M., Sotelino, A. y Lorenzo, M. (2015). Aprendizaje-servicio y misión civica de la universidad. Una propuesta de desarrollo. Barcelona, España: Octaedro.

Tapia, M. (2006). Aprendizaje y servicio solidario. En el sistema educativo y las organizaciones juveniles. Buenos Aires, Argentina: Ciudad Nueva.

Tünnermann, C. (2006). La educación superior en el umbral del siglo XXI. Caracas, Venezuela: CRESAL Unesco.

Universidad Católica Silva Henríquez [UCSH] (2014a). Modelo de Formación Universidad Católica Silva Henríquez. Santiago de Chile: UCSH.

Universidad Católica Silva Henríquez [UCSH] (2014b). Competencias genéricas $\operatorname{Res} N^{\circ} 054 / 2014$. Santiago de Chile: UCSH.

Universidad Católica Silva Henríquez [UCSH] (2016). Plan de Formación Integral. Santiago de Chile: UCSH.

Universidad Católica Silva Henríquez [UCSH] (2017). Guía procedimental. Competencias genéricas UCSH 2017. Santiago, Chile: UCSH.

Zabalza, M. (2007). La didáctica universitaria. Bordón 59 (2-3), 489-509.

Zabalza, M. (2012). Nuevos enfoques para la didáctica universitaria actual. Perspectiva 29(2), 387-416. https://doi.org/10.5007/2175-795X.2011v29n2 p387 